TITRE: LES PROBLÉMATIQUES DU RECRUTEMENT AUPRÈS DES PATIENTS ATTEINTS DE TRAUMATISMES CRANIOCÉRÉBRAUX (TCC) ET D’ALZHEIMER

Auteur(s): Kim Charest et Julie Bouchard

PublicATION: RECRUTEMENT ET CONSENTEMENT À LA RECHERCHE : RÉALITÉS ET DÉFIS ÉTHIQUES

PAGES: $115-126$

ISBN: 978-2-7622-0357-8

Directeurs: Ana Marin, BÉatrice Eysermann et Michel T. Giroux

URI: HTTP://HDL.HANDLE.NET/11143/14182

DOI: HTTPS://DOI.ORG/10.17118/11143/14182 


\section{Les problématiques du recrutement auprès des patients atteints de traumatismes craniocérébraux (TCC) et d'Alzheimer}

Kim Charest, étudiante au doctorat en neuropsychologie, Université du Québec à Montréal Julie Bouchard, Ph. D., directrice de doctorat en psychologie, neuropsychologue et professeure, Université du Québec à Chicoutimi

Résumé : L'expérimentation de nouvelles technologies d'assistance et d'intelligence artificielle s'avère nécessaire à l'augmentation de l'autonomie et de l'indépendance des populations vulnérables. Notamment, les patients ayant subi un traumatisme craniocérébral (TCC) et ceux atteints de la maladie d'Alzheimer sont susceptibles de pouvoir bénéficier de tels outils technologiques au quotidien. En raison des difficultés motrices, des problèmes cognitifs et des troubles de mémoire présents dans ces populations, les différentes étapes des recherches en sont complexifiées, particulièrement en matière de recrutement. Les expérimentations menées au Laboratoire d'intelligence ambiante pour la reconnaissance d'activités (LIARA) ont permis de souligner plusieurs problématiques éthiques liées à la recherche auprès de ces populations. D’autre part, ces études ont également mené à une panoplie de solutions simples et efficaces afin de travailler avec les proches, d'obtenir un consentement valable et d'éviter les enjeux liés aux problèmes cognitifs.

Mots clés : recrutement de participants, technologies d'assistance cognitive, consentement éclairé, troubles cognitifs. 


\section{Introduction}

Le recrutement de certaines clientèles pour la participation à un projet de recherche est en soi une expérience. Lorsque les participants visés par un projet ont des atteintes cognitives et/ou sont déclarés inaptes, faire preuve de créativité, autant pour le recrutement que pour la participation concrète, est essentiel. Les recherches qui sont effectuées au Laboratoire d'intelligence ambiante pour la reconnaissance d'activités (LIARA) font fréquemment appel à des participants ayant des atteintes cognitives puisqu'il s'agit des personnes principalement visées par le développement des technologies. Dans les dernières années, des patients atteints de traumatismes craniocérébraux (TCC) ainsi que ceux souffrant de la maladie d'Alzheimer ont pris part aux recherches effectuées. Les projets de recherche dans lesquels ces participants étaient impliqués avaient pour but d'évaluer l'efficacité de technologies d'assistance cognitive auprès de ces clientèles, considérées comme étant vulnérables. Comme le recrutement et la réalisation des expériences auprès de ces populations étaient souvent complexes, il semblait nécessaire de partager avec d'autres professionnels les expériences vécues, les différents obstacles ayant été surmontés ainsi que les stratégies ayant été développées. En plus de favoriser l'approfondissement des réflexions à propos des difficultés particulières vécues par ces patients et des défis quotidiens qu'ils doivent affronter, l'objectif est également de se pencher davantage sur la place qu'occupe l'éthique dans les recherches auprès de populations vulnérables et de réfléchir à des solutions accessibles et efficaces pour faciliter les étapes du recrutement et du consentement.

\section{Présentation}

Les travaux entrepris par la Chaire de recherche sur l'intelligence ambiante et les technologies d'assistance cognitive (CRIAAC), dont fait partie le LIARA, visent le développement d’outils technologiques spécialisés subvenant aux besoins des patients avec des problématiques neurologiques. Plus particulièrement, le but de l'équipe multidisciplinaire est de permettre une plus grande autonomie dans l'accomplissement des activités de la vie quotidienne (AVQ) ${ }^{1}$ de cette population. Il est important de comprendre que les troubles vécus par les participants visés par les recherches du LIARA sont souvent envahissants et restreignent les patients dans différentes sphères de leur vie, d'où l'intérêt d'augmenter leur autonomie et de diminuer leur sentiment de détresse face à cette situation.

Deux clientèles sont principalement visées au LIARA : les personnes avec un TCC et ceux ayant l'Alzheimer. Tout d'abord, un TCC survient chez une personne ayant subi un choc important à la tête provoquant d'importantes séquelles. Selon la force d'impact de ce choc, l'importance des saignements et la présence d'un coma ou non, la gravité du TCC est établie médicalement et peut se situer de léger à sévère (Paquette, 2016). Plusieurs phases suivent l'impact et peuvent constituer des visées pour certaines recherches. En effet, certaines études débutent alors que le client est dans le coma, ce qui requiert des façons de procéder particulières pour avoir le consentement, alors que d'autres se passent plus tard dans leur réadaptation (le cas de nos études) où le patient a déjà travaillé plusieurs aspects de récupération dans des centres hospitaliers et de réadaptation. Dans les études que nous avons réalisées, il a été nécessaire de travailler avec des participants ayant subi des TCC modérés ou sévères uniquement puisque, dans ces cas, les symptômes cognitifs et comportementaux subséquents étaient plus importants. Dans le cas des patients atteints de la maladie d'Alzheimer, les patients recrutés se situaient à différents niveaux dans l'évolution de la démence, mais l'ensemble d'entre eux souffrait de troubles mné-

1. Les activités de la vie quotidiennes (AVQ) sont les activités élémentaires accomplies dans la routine habituelle d'une personne autonome et indépendante. Par exemple, cuisiner, s'habiller et se laver sont considérées comme étant des AVQ (Société Alzheimer du Canada, 2016). 
siques. Il faut également savoir que la maladie d'Alzheimer fait en sorte que les personnes atteintes présentent une importante difficulté à effectuer les activités de la vie quotidienne (AVQ) et domestique (AVD). Aussi, il est possible d'observer, chez ces personnes, une difficulté à comprendre les notions abstraites, une diminution du jugement et une perte d'initiative (Société Alzheimer du Canada, 2016).

Les prototypes de technologies à l'essai sont donc créés dans l'intention de permettre à des clientèles vulnérables d'obtenir un niveau d'indépendance supérieur en les accompagnant dans la réalisation de différentes tâches et des AVQ, comme la cuisine, ou encore d'alléger la responsabilité des proches aidants et des intervenants². Ce laboratoire, situé dans les locaux de l'Université du Québec à Chicoutimi (UQAC), est en fait un prototype grandeur nature d'appartement intelligent doté de capteurs et d'effecteurs en tout genre. Les travaux effectués sont uniques et engendrent des défis de toutes sortes, qu'il s'agisse de l'aspect psychologique, informatique, physique ou, bien entendu, de l'aspect éthique.

\section{Défis liés à l'éthique de la recherche}

L'éthique en recherche est essentielle à la protection et au respect des participants. À cet effet, des règles et des démarches bien définies se doivent d'être respectées et suivies minutieusement. Malgré la nécessité incontestable d'un tel cadre, la situation des chercheurs demeure difficile puisque les projets de recherche se voient parfois contraints à plusieurs niveaux par ces conditions. Notamment, le recrutement s'avère parsemé d'embûches lorsqu'il est question d'éthique et de consentement libre et éclairé.

\section{Article 21 du Code civil du Québec et notion de risque minimal.}

D’abord, il est nécessaire d'évaluer convenablement la notion de risque attribué à la recherche présentée. Puisqu'il était question de travailler avec une clientèle étant jugée comme vulnérable et que certains des candidats sont en situation d'inaptitude, il était nécessaire de tenir compte des directives données par la Direction de l'éthique et de la qualité du Ministère de la Santé et des Services sociaux à l'effet que «les risques encourus lors de l'expérimentation ne doivent pas être hors de proportion avec les bénéfices espérés» (EPTC2, 2014; MSSS, 2016). Ce ne fut pas problématique à l'égard des projets menés par le CRIAAC, puisque les recherches effectuées comportaient un risque minimal pour l'intégrité des participants. Par contre, les modalités liées au consentement éclairé ont provoqué quelques difficultés. En effet, dans le cadre de la l'article 21 du Code civil du Québec, un participant considéré comme inapte aux yeux de la loi ne peut consentir par lui-même à participer à l'étude (Hébert, 2014). Autrement dit, un représentant légal doit être présent pour que le recrutement soit fait adéquatement.

L'enjeu soulevé réside dans le fait que les participants susceptibles de participer aux études demeurent, la plupart du temps, dans des centres spécialisés ou dans des hôpitaux. II n’y a donc pas toujours présence de tuteurs légaux ou de personnes habilitées à consentir aux soins lors du recrutement des personnes en situation d'inaptitude ou qui sont en évaluation à ce sujet. Cet élément a contraint les membres de l'équipe du CRIAAC à augmenter leurs efforts pour faire en sorte que le personnel des centres puisse rejoindre la famille. En effet, les règles d'éthique en place et les politiques des centres empêchent les chercheurs d'entrer directement en contact avec les participants potentiels (Hébert, 2013).

2. L'une des recherches en question avait pour but de vérifier l'efficacité d'une cuisinière intelligente lors de la réalisation d'une recette de pâté chinois comparativement à l'efficacité de l'assistance humaine. Dans un premier temps, les participants étaient guidés par la cuisinière, qui émet des sons et des indications programmées préalablement, et ensuite par une personne afin de comparer la quantité d'erreurs effectuées. 
À cet effet, les intervenants sont parfois contraints à effectuer une grande partie du recrutement à la place des chercheurs et des assistants. Bien que ceux-là manifestent généralement une grande volonté d'aider, la charge de travail immense dans leur emploi les rend moins enclins à prendre en charge cette responsabilité et ils sont souvent dans l'impossibilité d'expliquer complètement le projet de recherche (qu'ils connaissent plus ou moins) ainsi que les implications qui y sont liées. Pour leur part, les médecins, qui ont la possibilité d'offrir aux patients de prendre part aux projets de recherche, mettent souvent cette option de côté puisqu'il ne s'agit pas d'un aspect prioritaire pour la santé du patient et qu'ils sont conscients que les proches sont submergés dans les démarches à effectuer.

L'entourage des patients, surtout dans les cas d'inaptitude, peut également faire preuve de surprotection. Souvent eux-mêmes en période de choc et d'adaptation, les proches tentent, parfois de façon démesurée, de protéger la personne souffrante. Ceux-ci croiront donc que les modalités et les implications liées à la recherche seront trop exigeantes et complexes pour le patient et refuseront de signer le consentement. Pourtant, les risques associés à nos recherches sont minimaux, mais la surprotection des proches demeure prédominante. Chez les médecins, il est possible d'observer ce phénomène lors de l'annonce du diagnostic. Autrement dit, ils n'osent pas toujours suggérer la participation à un projet de recherche à ce moment puisqu'ils éprouvent de la compassion et présupposent que les patients et leurs proches ne sont pas en état d'entendre parler d'une recherche.

Ces situations font en sorte qu'il y a une grande partie de la population visée à laquelle nous n'avons pas accès ou que nous devons, malheureusement, exclure de l'échantillon. Il est nécessaire de noter que les patients inaptes ou en voie de le devenir font partie de la clientèle principale visée par le développement d'outils d'assistance cognitive. En les excluant à cause de la difficulté à communiquer avec le tuteur légal ou par non-transmission de l'information relative à la recherche, il y a une perte de données d'une valeur non négligeable. Ainsi, la forte majorité des participants aptes lors des expérimentations peuvent constituer une source de biais en ce qui concerne les résultats, puisqu'ils présentent des symptômes plus légers et réussissent donc à effectuer les tâches plus facilement (Voyer et St-Jacques, 2006).

Également, en ce qui a trait au consentement éclairé, le facteur lié aux difficultés de langage des participants est central. Les patients atteints de TCC ou de la maladie d'Alzheimer souffrent souvent d'altérations de la compréhension et de la production du langage, ce qui rend plus complexes la communication et la manifestation des besoins (Proulx, 2009). Ainsi, afin d'obtenir un consentement éclairé réel, il est nécessaire de prendre le temps d'expliquer correctement la nature des implications de la participation ainsi que de s'assurer de la compréhension complète et juste de l'entièreté de ces éléments, autant pour le participant que pour le proche l'accompagnant.

\section{Le recrutement}

Outre les défis liés directement aux principes éthiques, plusieurs obstacles associés aux déficits cognitifs et aux diverses craintes des participants surviennent lors des différentes étapes d'une recherche.

Tout d'abord, il est inacceptable de tenter de recruter des participants alors qu'ils sont encore en période de choc (post-annonce du diagnostic ou post-accident). Pour ce qui est des patients atteints de TCC, leurs symptômes sont plus sévères, voire plus aigus, dans un certain délai suivant leur accident. II n'est pas rare de voir les gens atteints de TCC faire des progrès dans les mois suivants leur traumatisme. Bien que les répercussions soient nombreuses et marquantes, une récupération partielle, voire quasi totale dans de rares cas, est possible 
pour certains (Gil, 2014). Il pourrait être intéressant de les recruter durant la période suivant l'accident afin de faire l'expérimentation alors que les symptômes sont importants, mais il ne serait pas très éthique de s'inclure dans cette période intense de réadaptation et d'adaptation à la condition.

Également, le fait de ne pas pouvoir profiter des technologies étudiées dans les projets de recherche posait problème chez certains candidats approchés ou chez leurs proches. Puisque les outils conçus par l'équipe du CRIAAC ne pourront être utilisés que dans un temps indéterminé, certains mentionnaient ne pas vouloir participer par crainte de perdre leur temps ou par désintérêt des objectifs à long terme de ces projets. Ce phénomène est demeuré présent même s'il a été question de miser sur l'altruisme des participants potentiels. En effet, bien qu'un accent ait été mis sur les développements dont bénéficieront les générations futures lors des explications, le manque d'intérêt demeurait palpable puisqu'ils ne seraient pas eux-mêmes en mesure de profiter des avancés technologiques espérés. II aurait peut-être été préférable de soulever les éléments attrayants de la participation, puisqu'au final la majorité des participants qui ont accepté de prendre part aux expérimentations ont souligné leur satisfaction face à leur participation. Par exemple, certains d'entre eux ont mentionné avoir passé «du bon temps en bonne compagnie», qu'ils voyaient cela comme une «activité différente» et étaient heureux d'avoir pu parler de ce qu'ils vivaient.

Également, des craintes liées à l'utilisation des outils d'assistance cognitive étaient notables chez les patients atteints de la maladie d'Alzheimer. Ceux-ci éprouvaient beaucoup de réticence à travailler avec des outils technologiques. Puisque les gens atteints de la maladie d'Alzheimer sont souvent plus âgés, ils sont moins enclins à apprendre à travailler avec de nouveaux outils, surtout lorsqu'il s'agit du domaine technologique avec lequel ils ne sont pas familiers (Verreault, 2018).

Un autre motif qui était invoqué était le fait de ne pas pouvoir être utile en raison de leur déficit, même si la situation était tout à fait l'inverse. En raison des atteintes cognitives dont elles souffrent, ces personnes sont malheureusement parfois dans l'incapacité d'apprendre et de retenir de nouveaux éléments. Lorsqu'ils sont conscients de ce fait, les patients ont parfois la certitude de ne pas être assez compétents pour prendre part à des recherches universitaires. Ils refusent donc parfois de consentir, se sentant inutiles ou inadéquats. Pourtant, c'est principalement parce qu'ils ont ces déficits qu'ils sont intéressants aux yeux des chercheurs. Ces croyances sont évidemment erronées, mais il est fondamental de rassurer les patients à ce sujet une fois le contact établi avec eux.

\section{Contexte de réalisation des études}

Des études menées par le Centre d'études sur le stress humain (CSEH) démontrent que le fait de tester les capacités mnésiques ou cognitives des personnes âgées dans un environnement qui leur est défavorable a un impact négatif sur leur performance. Notamment, ces études révèlent que le temps entre la prise de rendez-vous et la rencontre elle-même, les modalités associées au transport et l'environnement médical peuvent créer de l'anxiété chez les personnes âgées lorsqu'il est question de tests. En effet, le CSEH démontre l'importance que joue le rôle de l'environnement dans les performances aux tests lorsqu'on compare les résultats obtenus à ceux de plus jeunes candidats (Lupien et coll., 2012).

Ces facteurs ont également été observés par l'équipe du CRIAAC. Il va de soi que la question du transport est plutôt anxiogène pour les gens dans ces situations. D'une part, s'ils sont inaptes ou qu'ils ne peuvent pas conduire, le fait de devoir trouver quelqu'un pour les accompagner ou d'organiser un transport adapté peut les angoisser et même empêcher leur consentement à participer. D'autre part, s'ils conduisent, le fait de devoir se 
rendre à l'université, de trouver un stationnement et de payer l'horodateur peut provoquer le même sentiment et, donc, une diminution de leur performance ou de leur désir de participer (Lupien et coll., 2012).

Lorsqu'on effectue des recherches dans les murs de l'université, il faut se souvenir qu'il représente un lieu jeune et dynamique dans lequel les personnes plus âgées se sentent plus ou moins à l'aise. Ainsi, ils ne seront pas attirés à participer à une étude se déroulant à cet endroit. Il est important aussi de souligner qu'il s'agit d'un lieu vaste avec plusieurs édifices et étages, ce qui peut poser problème chez les patients souffrant de la maladie d'Alzheimer puisqu'ils sont victimes d'une importante désorientation dans l'espace. Ils ont donc peur de se perdre dans les différents locaux et de ne trouver personne pour les aider à s'orienter convenablement. Lorsqu'ils ne peuvent être accompagnés par un proche pour diverses raisons, ces craintes sont accentuées. Dans la vie de tous les jours, cette désorientation constitue un important facteur d'isolement, de surveillance et de risque de fugue ou d'égarement (Ruis, 2008).

\section{Solutions \& Astuces}

Face aux nombreuses situations énumérées précédemment, l'équipe de recherche du CRIAAC n'avait guère d'autres choix que de faire preuve de créativité afin de poursuivre ses démarches. Bien qu'il ne soit pas possible d'éliminer totalement les éléments problématiques au recrutement des participants, il fut question d'utiliser différentes approches et méthodes dans le but de faciliter cette étape ou, du moins, d'en réduire les obstacles. Les caractéristiques particulières des patients ainsi que les règles strictes (mais logiques pour protéger les participants) de l'éthique en recherche ont fait en sorte que des solutions qui facilitaient à la fois le rôle des chercheurs mais également celui des participants ont été développées. En effet, plusieurs des solutions proposées permettaient aux participants d'être moins anxieux et de se sentir à l'aise en ce qui concernait le processus de consentement et amélioraient donc leur participation.

\section{Recrutement}

On ne peut passer sous silence le sujet du travail interdisciplinaire plutôt complexe qui s'impose lors d'une recherche auprès de patients vulnérables. Puisque la collaboration avec les centres et les hôpitaux est obligatoire pour le recrutement auprès de ces clientèles, le fait d'avoir une relation professionnelle de bon terme avec les intervenants est un atout. Il faut comprendre leur situation et se montrer empathique à leur égard, tout en s'adaptant à leur rythme de travail. Ainsi, ils se sentiront respectés et auront davantage envie d'aider dans le processus de recrutement. Travailler en étroite collaboration avec les intervenants et les centres permet d'approcher les participants de façon plus personnalisée, d'organiser des services mieux adaptés, d'être supporté tout au long du processus et de profiter d'une plus grande accessibilité dans le réseau. Bien souvent, les infirmières ou travailleurs du milieu connaissent leurs patients, leurs réactions, leurs inquiétudes et leurs difficultés particulières. Ainsi, travailler ensemble vers l'objectif commun d'assurer le confort et le bien-être de la personne lors de sa participation à la recherche est un aspect considérable. Il peut s'agir aussi, pour des membres de l'équipe de recherche, d'aller passer des journées dans des résidences, des centres - parfois même des fins de semaine - ou dans des événements pour avoir l'occasion de se faire connaître, d'avoir une table pour parler de la recherche où les proches peuvent venir poser des questions et voir les outils utilisés.

Il semble pertinent, lors de l'approche d'un participant, de mettre l'accent sur le but visé par la recherche et sur ses avantages. En expliquant les raisons pour lesquelles de tels projets sont mis en place et en spécifiant les bénéfices espérés, les gens ont tendance à développer de l'intérêt pour le projet en question et à s'intéres- 
ser aux avancements possibles dans le domaine. Bien souvent, les patients ont de la difficulté à s'imaginer concrètement les bénéfices des recherches effectuées et ce qu'il est possible de produire ou d'améliorer avec les résultats. Ainsi, en leur expliquant de façon précise les objectifs visés et les avantages qui en découlent, ils deviennent curieux à ce sujet et semblent plus enclins à participer aux recherches.

\section{Consentement}

Préciser les tâches à accomplir, se montrer clair et concis envers les participants quant à leurs responsabilités, souligner que leurs déficits ne sont pas problématiques pour le déroulement du projet de recherche peut faciliter l'approche vers le consentement. Précisément à cause de leurs déficits, les participants sont parfois réticents puisqu'ils sont conscients de leurs lacunes. Par exemple, les patients ayant vécu un TCC souffrent d'atteintes cognitives, c'est-à-dire que leur mémoire, leurs fonctions attentionnelles et exécutives, leur langage, leur capacité de planification ainsi que d'autres facultés peuvent être altérés (Paquette, 2016). Aussi, ils peuvent se retrouver dans l'incapacité d'apprendre de nouveaux éléments ou encore de comprendre les étapes successives nécessaires à la réalisation d'une nouvelle activité. La conception d'une recette, par exemple, peut constituer un défi de taille pour une personne ayant subi un TCC puisque les parties du cerveau nécessaires dans la planification et la rétention des diverses étapes sont généralement touchées (Bolduc, 2015).

Il est donc évident que le fait de prendre son temps et d'être patient avec eux les rassure énormément. En leur mentionnant comment l'équipe de recherche compte s'y prendre pour les aider dans les démarches liées à la participation, comme les modalités de transport et l'accueil à l'université, ils se sentiront compris et plus à l'aise. Il faut aussi tenir compte du fait que peu de gens désirent participer lorsqu'ils ne sont pas mis au courant de ce qui les attend exactement, il est donc essentiel d'avoir un discours concret et vulgarisé. Également, il va de soi que d'énumérer précisément les actions qu'ils devront effectuer lors de l'expérimentation diminue grandement les craintes et l'anxiété liées à la recherche. Une peur quant à leurs capacités physiques peut être présente due au fait que ces personnes peuvent être aussi aux prises avec difficultés motrices. Notamment, pour les patients ayant subi un TCC, l'équilibre et la coordination peuvent être affectés à différents degrés. Ceci peut évidemment causer des chutes, des blessures importantes et des diminutions d'efficacité dans la réalisation des activités quotidiennes (Dault, 1998). Il faut donc bien les renseigner sur la sécurité pour eux lors de la réalisation de l'expérimentation. Bien souvent, lorsque les actions à effectuer sont claires et bien détaillées, ils comprennent que les étapes à accomplir sont plutôt simples et qu'ils sont en mesure de les réussir contrairement à leur croyance initiale.

Afin d'avoir un consentement éclairé valide, il est nécessaire que les futurs participants comprennent bien le rôle qu'ils joueront et les implications auxquelles ils devront répondre. Ainsi, le fait d'adopter un langage familier est un atout qui facilite la création d'un lien de confiance entre le chercheur et le participant. Il va de soi qu'en adoptant un langage trop spécifique lié à un champ d'expertise le chercheur risque de perdre l'attention du participant ou encore de lui faire sentir qu'il est incapable d'accomplir la tâche demandée. Ainsi, un langage familier et plus vulgarisé peut détendre l'atmosphère et favoriser la communication. De plus, puisque les patients approchés dans le cadre des projets du CRIAAC présentent régulièrement des difficultés de langage, le fait d'adapter son discours au niveau de l'interlocuteur a un effet positif non négligeable. Également, en ce qui concerne le contact à établir avec le participant, il faut savoir tenir compte des caractéristiques comportementales liées aux atteintes neurologiques (RAPTCCQ, 2016). En effet, il est courant de voir des changements dans le comportement, des désinhibitions ou des changements d'humeur rapides. Il faut savoir écouter les participants, les encadrer avec douceur, être patient et, surtout, respecter leur rythme et entendre les émotions que 
peuvent susciter les expérimentations (autant pour les participants que pour les proches) tout en les référant au besoin à des ressources adaptées.

Selon les expériences vécues par l'équipe de recherche du CRIAAC, mettre l'accent sur le côté humain de la recherche est indispensable lors du recrutement comme tout au long du processus d'expérimentation (Wilkinson, 2002). En effet, le chercheur se doit d'être à l'écoute des besoins, des craintes et des attentes de ses participants, surtout lorsqu'il s'agit d'effectuer le recrutement et de passer à l'étape du consentement. En tant que chercheurs auprès de clientèles vulnérables, il est nécessaire de se mettre à la place des participants et de tenter de comprendre leur situation. Le fait de se questionner sur ce qui peut leur causer de l'anxiété ou les placer dans une situation inconfortable demeure essentiel, car, après tout, la recherche a pour objectif de servir les individus atteints.

L'un des éléments qui semblent avoir facilité l'intégration des participants à la recherche est l'éducation à la technologie. Puisque les technologies d'assistance cognitive sont peu connues et qu'elles engendrent de l'anxiété chez la clientèle visée, il est primordial d'en démontrer l'utilité et de permettre au participant d'être plus familier avec cette technologie avant d'entreprendre l'expérimentation. Comprendre le fonctionnement de ces machines et savoir comment les utiliser diminuera, voire éliminera, considérablement leur méfiance initiale. Aussi, il est favorable de mentionner ou de rappeler les bénéfices de l'avancement des technologies d'assistance cognitive et ce que ces prototypes pourront apporter à la vie quotidienne des gens qui y auront recours. Il ne faut pas oublier de souligner le fait qu'ils n'auront pas à gérer le fonctionnement des technologies, c'est-à-dire qu'ils ne sont pas en contrôle gadgets inconnus et de prototypes complexes. Leur tâche demeure de réaliser des activités semblables à la vie quotidienne, ce qui les rassure énormément.

Ensuite, puisque l'organisation du transport est très anxiogène chez ces patients, il est idéal de diminuer le nombre de passations. Même si, dans le cadre de certaines études, il est parfois obligatoire que les participants se déplacent à plusieurs reprises, il est préférable de réduire ce facteur. Par exemple, dans le cadre d'expérience requérant deux passations, le fait d'accorder un temps de repos allongé peut permettre à la personne de se déplacer une seule fois plutôt que deux. Cet élément peut sembler anodin, mais il peut faire une grande différence dans le recrutement. Alors que certains refuseront de participer puisqu'ils s'inquiètent des modalités de transport, les participants à qui l'alternative de diminuer le nombre de séances sera proposée seront plus enclins à consentir à participer.

D’un côté plus pratique, l'utilisation d'un système de rappel est un outil très apprécié par les participants. Puisque les clientèles visées par ces projets sont sujettes à éprouver des difficultés mnésiques et de la désorientation dans le temps (Ruis, 2008), cette astuce permet d'éviter l'oubli de rendez-vous. Par exemple, lors de la première phase expérimentale auprès des participants atteints d'Alzheimer, $25 \%$ de ces derniers ne se sont pas présentés au rendez-vous fixé puisqu'ils avaient oublié l'entente prise préalablement. Ainsi, il fut jugé pertinent de mettre en place un système de rappel par lequel il fut possible de joindre les participants, et ce, jusqu'à trois reprises. Depuis l'implantation de ce système, aucun oubli de rendez-vous n'a été rapporté par l'équipe de recherche (Lapointe, en rédaction).

Finalement, offrir une compensation aux participants pour les remercier de leur implication dans un projet peut être une source de motivation. Évidemment, les compensations monétaires semblent être les plus attirantes pour les participants. Par contre, puisque les budgets ne permettent pas toujours aux chercheurs d'offrir ce genre de récompenses, il fut question de se tourner vers une autre solution. Ainsi, un guide d'outils fut créé dans le but d'offrir une compensation accessible et utile à la clientèle (CRIAAC, 2015). Ce guide d'outil est en quelque sorte un magazine dans lequel il est possible de trouver différentes adaptations déjà existantes qui 
permettent de compenser pour plusieurs aspects de la vie quotidienne, et qui précise à quelle clientèle l'adaptation spécialisée convient de manière plus précise. Par exemple, il est possible d'y trouver des modèles de robinet à technologie DST, de plaque de cuisson à induction, d'aspirateur robot, de porte-clés siffleur, etc. De cette façon, les gens atteints d'Alzheimer ou de TCC se voient dans la capacité de trouver facilement des objets qui leur procureront une plus grande autonomie. Dans bien des cas, ce guide d'outil fut grandement apprécié.

\section{Conclusion}

À la lumière des enjeux soulevés, il est vrai de dire que la recherche auprès des populations vulnérables demeure un domaine parsemé de défis et de surprises. Bien que les règles d'éthique soient claires et préalablement définies, le passage à l'étape du recrutement et de consentement se voit complexifié par ces règles ainsi que par les restrictions qu'imposent les normes juridiques. En plus de cela, le travail auprès de clientèles vulnérables est plus difficile en raison même des problématiques particulières vécues par ces patients. Une fois la tête plongée dans le travail de recherche, il devient parfois difficile de poursuivre ses objectifs tout en respectant à la lettre chacun des principes éthiques, d'où l'insatisfaction de certains chercheurs. Toutefois, selon les expériences vécues par l'équipe du CRIAAC, en faisant preuve de créativité et en s'harmonisant aux conditions présentées, il demeure possible pour les chercheurs d'arriver à leur fin. En somme, il reste primordial de garder à l'esprit que, peu importe l'angle avec lequel ce sujet est abordé, tous tentent de travailler pour le bien des patients et pour optimiser leur condition. Que notre rôle soit celui d'assurer leur protection par l'entremise de l'éthique ou bien celui de développer les connaissances afin d'améliorer leur sort, l'essentiel demeure de conserver ces participants au centre de nos motivations et de nos efforts. 


\section{Bibliographie}

Bolduc, F. (2015). Effets du traumatisme cranio-cérébral léger chez la personne âgée sur les processus mnésiques et exécutifs, en lien avec les habitudes de vie (thèse de doctorat, Université Laval, Québec, Canada).

Chaire de recherche sur l'intelligence ambiante et les technologies d'assistance cognitive (CRIAAC). (2015). Guide d'outils: Services offerts à la population oeuvrant près d'une personne en perte d'autonomie, Repéré à http://evalorix.com/wp-content/uploads/2015/06/561-Services-offerts-population-perte-dautonomie.pdf.

Dault, M. (1998). Réduction motrice chez des sujets ayant un traumatisme cranio-cérébral (mémoire de maîtrise, Université du Québec à Trois-Rivières, Trois-Rivières, Canada).

Conseil de recherches en sciences humaines du Canada, Conseil de recherches en sciences naturelles et en génie du Canada, Instituts de recherche en santé du Canada. (2014). Énoncé de politique des trois conseils: Éthique de la recherche avec des êtres humains. (cité dans le texte comme EPTC2). Repéré à http://www.ger.ethique.gc.ca/pdf/fra/eptc2-2014/EPTC_2_FINALE_Web.pdf

Gil, R. (2014). Neuropsychologie, (6eédition). France : Elsevier-Masson.

Hébert, A-M. (2013). Modifications à l'article 21 du Code civil du Québec : enfin une bonne nouvelle pour les chercheurs et les comités d'éthique de la recherche. Consortium national sur l'intégration sociale, 05(1). Repéré à https://oraprdnt.uqtr.uquebec.ca/pls/public/gscw031?owa_no_site=4958\&owa_no_ fiche=182\&owa_bottin=

Lapointe, J. [thèse en rédaction]. Département de psychologie, Université du Québec à Chicoutimi.

Lupien, S., Sindi, S. et Wan, N. (2012). Quand on teste, est-ce qu'on stresse? Centre d'études sur le stress humain (CESH), Repéré à http://www.stresshumain.ca/stress-et-vous/stress-chez-les-aines/quand-onteste-est-ce-quon-stresse/

Ministère de la santé et des services sociaux du Québec (MSSS). (2016). Recherche portant sur des personnes mineures ou majeures inaptes, Repéré à http://ethique.msss.gouv.qc.ca/lethique-en-bref/recherche-aupres-des-personnes-mineures-ou-inaptes.html

Paquette, C. (2016). Traumatisme cranio-cérébral. Association québécoise des neuropsychologues. Repéré à https://aqnp.ca/documentation/neurologique/le-traumatisme-cranio-cerebral/

Proulx, G. (2009). «Les déficiences cognitives et démence», Société canadienne de psychologie. Repéré à http://www.cpa.ca/lapsychologiepeutvousaider/deficiencescognitivesetdemence/

Regroupement des associations de personnes traumatisées craniocérébrales du Québec (RAPTCCQ). (2016). «Le TCC, c'est quoi?», Repéré à http://www.raptccq.com/raptccq/le-tcc-cest-quoi.html

Ruis, J. (2008). Désorientation temporo-spatiale et déambulation, comment accompagner au mieux? Revue de l'infirmière, 143, 6

Société Alzheimer du Canada (2016) La maladie, 10 symptômes précurseurs. Repéré à http://www.alzheimer. $\mathrm{ca} / \mathrm{fr} /$ About-dementia/Alzheimer-s-disease/10-warning-signs

Verreault, A. (2018). Étude exploratoire sur l'attitude et les habitudes d'utilisation à l'égard de la technologie, par les personnes atteintes de la maladie d'Alzheimer, de leurs proches aidants et des intervenants travaillant auprès d'eux (thèse de doctorat, Université du Québec à Chicoutimi, Chicoutimi, Canada). 
Voyer, P. et St-Jacques, S. (2016). L'article 21 du code civil et la recherche auprès des aînés atteints de démence dans les milieux de soins de longue durée au Québec : une analyse, un constat et une proposition. Conseil des aînés du Québec, Repéré à https://www.fsi.ulaval.ca/sites/default/files/documents/pdf/Personnel/Philippe\%20Voyer/article_21_voyer_st-jacques_et_partenaires.pdf

Wilkinson, H. (2002). Including people with dementia in research. Dans Wilkinson H. (ed.) The perspectives of people with dementia: Research methods and motivations (pp. 9-25), London and Philadelphia: Jessica Kingslay Publishers. 